\title{
Usefulness of WhatsApp for Discussing Difficult Cases in Pathology Practice: A Moroccan Experience
}

\author{
Amal BENNANI' (D), Mohammed SEKAL² (D)
}

Department of Pathology, 'Mohamed I University, OUJDA, MOROCCO, ${ }^{2}$ Mohamed Benabdellah University, FES, MOROCCO

\begin{abstract}
Objective: The primary aim of the study was to evaluate whether the WhatsApp application can be used for obtaining a quick second opinion on histopathological and cytological diagnosis and also for discussing difficult cases in pathology practice.

Material and Method: A WhatsApp group named "FESPATH" was created with total of 17 pathologists (the authors of this manuscript as group administrators and 15 general pathologists from 7 different cities in Morocco, working in 12 different institutions as members). The group was used to discuss difficult routine sign out cases, to obtain a second opinion. Pathology-related academic files, books and links were also shared. At the end of 20 months, members were asked to complete a feedback questionnaire.

Results: Over a 20-month period, 86 cases were discussed with 515 posted pictures. 78 cases were related to histopathology, and 8 cases to cytopathology. Twenty-one links regarding educational materials and books were also shared. A total of 14 participants out of 17 were active participants, and the majority of them found the discussions very useful for overcoming challenging cases.
\end{abstract}

Conclusion: Sharing microphotographs of histopathological or cytological cases via WhatsApp is a very easy and fast method to obtain a second opinion in pathology practice and also to discuss difficult cases.

Key Words: Pathology, Smartphone, Second opinion, WhatsApp, Social media

\section{INTRODUCTION}

Technological improvements in the smartphone industry and social networking have changed our life. Social media has become a part of daily life for most people and is indispensable for communication between peers across the world (1). It has also increasingly shaped our professional life, as in pathology.

Pathology is a medical specialty, that involves the diagnosis of disease through microscopic examination of surgically removed organs, or biopsies. Pathologists need to have valuable knowledge and skills to reach a correct diagnosis. When diagnostic challenges persist, they have to consult other colleagues for a second opinion. The slides or blocks are usually sent to another pathology center for the second opinion. However, this practice usually leads to diagnostic delay, which can be critical for malignant tumors (2).

WhatsApp (WhatsApp, Inc., Mountain View, California, USA) is one of the most popular freeware instant messaging applications on smartphones. This application allows sending text messages, documents, images, user location, audio and video calls using an internet connection.

(Turk Patoloji Derg 2019, 35:134-138)

Received : 07.06.2018 Accepted : 15.09.2018
In addition, configuration of the smartphone camera with high resolution and zoom has made it possible for pathologists to take good quality images from any microscope showing the morphological details of the case with high accuracy. As most smartphones provide high-speed $4 \mathrm{G}$ or $5 \mathrm{G}$ internet access, images can be sent immediately via WhatsApp. As a result, the pathologist can obtain a second opinion and discuss difficult cases quickly and efficiently.

The aim of the current study was to determine the effectiveness of the WhatsApp application in the field of pathology for obtaining a second opinion and discussing difficult cases.

\section{MATERIAL and METHODS}

A WhatsApp group named "FESPATH" was created with a total of 17 pathologists (the authors of this study as group administrators and 15 general pathologists from 7 different Moroccan cities, working in 12 different institutions as members). All these pathologists had graduated from the Mohamed Benabdellah University in FES, a city in northern inland Morocco . The group was used to discuss difficult cases from sign out routine, and to obtain a second opinion.

Correspondence: Amal BENNANI

Bp 6210. Assaada 60020 OUJDA, MOROCCO

E-mail: bennaniamal@gmail.com Phone: +21 2662781319 
It was also used to share helpful links, charts, algorithms, figures from articles and updated classifications or books.

After a period of 20 months (from July 2016 to February 2018), members of the group were asked to complete a feedback questionnaire. The discussion time was calculated from the time of sending the photos and the time of the first response. The average response time and the average shared microphotograph was calculated using Excel.

Other statistics such as number of cases discussed, total number of posts, the number of cases by pathology, and total number of active participants were also calculated. An active participant was defined as a member who had more than two posts during this period. The group continues to discuss cases and share files to this day.

All cases were related to histopathology or cytopathology, and most of them were routine sign out cases. A brief clinical summary, radiological and macroscopic pictures accompanied by representative microscopic images were shared. In most cases, the microphotographs were taken directly from the microscope with a smartphone. Only one pathologist used a commercially produced adapter (Figure 1 ). At least one scanner view (x50), and 1 low power (x100) and 2 or more high power magnification $(\mathrm{x} 400)$ images were shared (Figure 2A-D)

The main thrust of the group was on sharing images of difficult cases to create discussion on the differential diagnosis and diagnostic pitfalls.

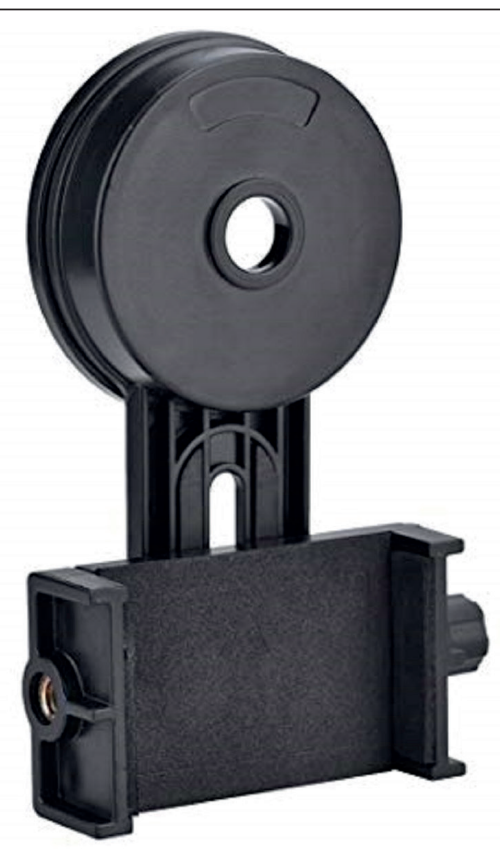

Figure 1: An example of a microscope adapter for smartphone used in our study.

\section{RESULTS}

Over a 20 -month period, 86 cases were discussed with 515 microscopic images shared. 78 cases were related to histopathology, and 8 cases to cytopathology (Table I,II). 20 links regarding online lectures and educational materials were also shared. A total of 14 participants out of the 17 were active participants. The average time required for the first discussion was $169.4 \mathrm{~min} \pm 78 \mathrm{~min}$. The average number of shared microphotographs was $4 \pm 2$ microphotographs.

In $71 \%$ of the cases (61 cases) the final diagnosis was resolved after discussion of the members of our group, while in 29\% (25 cases) the final diagnosis depended on ancillary techniques (immunohistochemistry, special stain) or an expert opinion.

The members of this group (excluding the authors of this paper) responded to the questionnaire (Table III). Most members liked this experience and found the discussions very useful for overcoming challenging cases. Regarding other social media applications, 50\% would like to use them for discussing cases and $30 \%$ were not sure about it.

Table I: Number of cases by pathology (n, \%)

\begin{tabular}{lc}
\hline Subspecialties covered in the study & $\begin{array}{c}\text { Number of cases } \\
(\mathbf{n}, \%)\end{array}$ \\
\hline Gastrointestinal pathology & $20(23 \%)$ \\
\hline Dermatopathology & $22(25 \%)$ \\
\hline Cytopathology & $8(9 \%)$ \\
\hline Gynecopathology & $17(20 \%)$ \\
\hline Soft tissue and bone pathology & $6(7 \%)$ \\
\hline Hematopathology & $4(5 \%)$ \\
\hline Pulmonary pathology & $4(5 \%)$ \\
\hline Salivary gland and thyroid & $5(6 \%)$ \\
\hline
\end{tabular}

Table II: Data of the use of WhatsApp by the group FESPATH over the study period of 20 months

\begin{tabular}{lc}
\hline Parameters & Number (n) \\
\hline Number of participants & 17 \\
\hline Number of cases posted & 86 \\
\hline Cases of histopathology & 78 \\
\hline Cases of cytopathology & 8 \\
\hline Number of active participants & 14 \\
\hline Passive participants & 3 \\
\hline Total number of posted images & 515 \\
\hline Links shared & 10 \\
\hline Files shared & 11
\end{tabular}


Table III: Questions asked to the participants and their responses

\begin{tabular}{lc}
\hline Questions & Response $\%$ \\
\hline 1- The use of WhatsApp is very important in work-related activities & $100 \%$ agree \\
\hline 2- First impression on creation of the Whats App group & $80 \%$ not sure, $20 \%$ excited \\
\hline 3- Did you find the discussions useful? & $60 \%$ useful, $40 \%$ very useful \\
\hline 4- Did you follow the discussions being done? & $75 \%$ most, $16 \%$ all, $9 \%$ some \\
\hline 5- Annoyance by numerous posts & $42 \%$ none, $58 \%$ sometimes \\
\hline 6- Did it help in building healthy interpersonal relationships & $100 \%$ yes \\
\hline 7- Did you feel that patient privacy was being compromised at any point? & $100 \%$ no \\
\hline 8- If discontinued, would you miss it? & $83 \%$ yes, $17 \%$ no \\
\hline
\end{tabular}

9- Given an option, would you like to use any other social media (such as Facebook) for interaction?

$50 \%$ yes, $30 \%$ may be, $20 \%$ no

10-Quality of the microphotographs

11-Did you like the links and shared files?

92\% good, $8 \%$ excellent

12-Did you think that this discussion resolved the diagnosis?

$100 \%$ yes

$50 \%$ most times, $50 \%$ sometimes
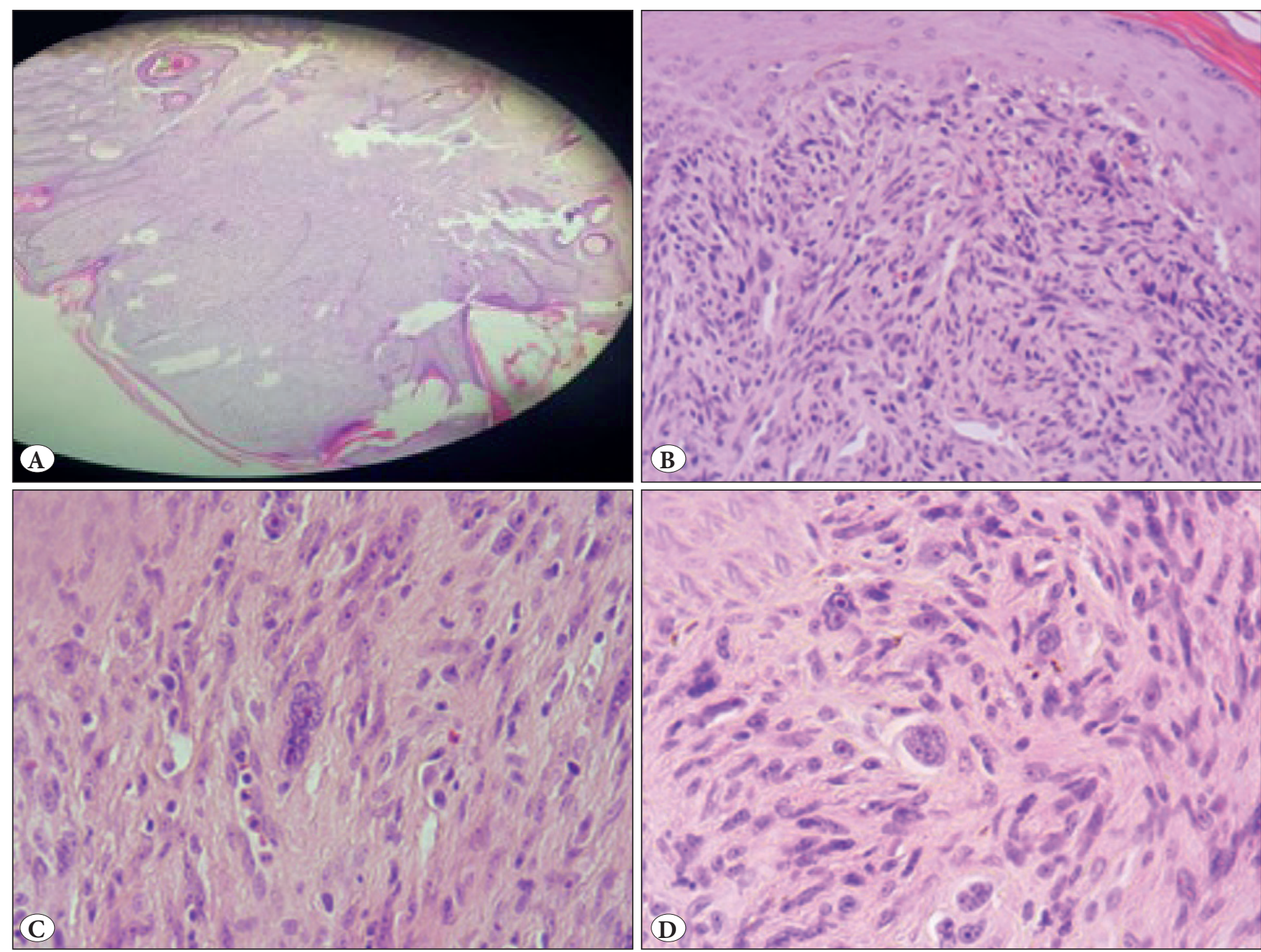

Figure 2: A case of atypical fibroxanthoma shared in the group Fespath. A) With a scanner view (H\&E; x50). B) Low power magnification (H\&E; x100). C-D) High power magnifications (H\&E; x400). 


\section{DISCUSSION}

Social media has definitely transformed our personal life. It has made communication easier, faster and more efficient than ever. With billions of users, Facebook, Twitter, WhatsApp and many others are wide-ranging and powerful social media platforms (1).

Recently, many papers have been published about the usefulness of the WhatsApp platform in medical practice (3-6). Most of them are about medical education, communication with patients, or discussion of the patients between doctors of many clinical branches (3). However, there are only a few studies about pathology and WhatsApp in the literature.

Petruzzi and De Benedittis concluded in their study that WhatsApp can be used as a telemedicine program for improving medical consultation and clinical examination in oral pathology (6). Zotti et al. reported that WhatsApp is an effective tool for improving oral health status during orthodontic multibracket treatment of adolescent patients (5).

Furthermore, Khanna et al. showed that WhatsApp is very useful for communication and improvement of patientrelated awareness, and also for orthopedic residents (4).

In pathology, Sarode et al. reported their experience with WhatsApp in obtaining a second opinion on the histopathological diagnosis in oral pathology practice (2). They studied 247 cases of 34 different oral pathologies that were photomicrographed using a smartphone through microscopes and then sent for a second opinion to 20 different oral pathologists by WhatsApp. They proved the effectiveness of the WhatsApp application in obtaining a fast second opinion for histopathological diagnosis

Moreover, a second experience in pathology was reported by Goyal et al. (7). A WhatsApp group was created to discuss interesting cases, and pathology-related academic issues. 16 cases were shared with 647 posts, in a period of 4 weeks. This group has increased interest in the specialty, particularly among the 1st year postgraduates, and will improve the academic performance of students if it is used for a long period.

In the current study, the main aim was to evaluate a new tool to discuss difficult routine sign out cases and to obtain a second opinion which is rapid and in real time. In $71 \%$ (61) of the cases, the final diagnosis has been resolved after discussion of the members of our group, while in $29 \%$ the slides or microphotographs of the cases were sent for expert opinion in Morocco or abroad. The time required for a second opinion ranged from 4 to 480 minutes, which is by far faster than a traditional request for second opinion.

The responses to the questionnaire were very encouraging. The majority of the participants of our WhatsApp group found the discussions very useful, but it can sometimes disturb the daily routine. This can be managed if we choose the right time for sharing cases like in the early morning when most of the pathologists are on their way to work, or around lunchtime and the evenings.

It is admitted that the resolution of images is decreased by the WhatsApp application. In the present study, most pathologists did not find any difficulty in the interpretation of the photomicrographs but in some cases asked to have more microphotographs with better quality in some areas of the slide.

Regarding other social media platforms, 50\% would like to use them for discussing interesting or challenging cases and $30 \%$ were not sure about it.

Twitter (Twitter, Inc., San Francisco, California, USA) and Facebook (Facebook, Inc., Menlo Park, California, USA) are other social media tools commonly used in medical practice (8-10). Facebook has made significant contribution in the field of pathology. Both trainees and pathologists in regular practice can use public professional Facebook pages, or pathology-related groups to improve their knowledge and to discuss difficult cases. Numerous pathology-related Facebook groups have been created such as Dermatopathology (http://bit.ly/1L7n5Yg), Bone and Soft Tissue Pathology (http://bit.ly/1EHYJQG), McKee Derm (http://bit.ly/2kfkKXT), Surgical (Anatomic) Pathologists (http://bit.ly/2xajmqC), and many others (11, 12)

Furthermore, Twitter can be used by pathologists to (1):

- Share images of challenging pathology cases.

- Share algorithms and links to scientific papers.

- Announce pathology courses or meetings.

- "Live-tweet" from conferences or meeting.

- Create short surveys using the native Twitter survey function.

WhatsApp is a good tool for discussing cases in histopathology and cytology as it allows quick answers and instantaneous discussion compared to other social media tools. It also provides more protection for patient information or data since WhatsApp groups are restricted to a number of pathologists and can be protected with a 
password. Consequently, there is no ethical violation. As well as using text messages to provide answers for shared cases, pathologists can also use voice messages to give their opinion. On the other hand, WhatsApp does not allow creating surveys or provide statistics of shared cases or other items like Twitter.

Sharing images on social media has been a cause of worry for doctors as it raises some possible ethical issues about patient privacy. However, most pathology images do not reveal patient identity. There are few basic rules that pathologists need to follow:

- It is judicious not to give the exact age of the patient and if possible to round it off to the nearest multiple of 5 .

- Do not mention the geographic subdivision of the patient

- Do not mention laterality wherever possible.

- Do not post full facial images. If they are necessary, you have to provide consent from the patient.

But in all cases, sharing pathology images for discussing or educational purposes on social media is legal and ethically acceptable even without patient consent (13)

Even though all social media applications are used to get a second opinion in pathology, it is important to emphasize that the ultimate responsibility belongs to the pathologist who signed out the pathology report.

In conclusion, the present study showed that WhatsApp is a good tool for discussing or obtaining a quick and instantaneous second opinion in challenging cases. Its daily and consistent use will tremendously enrich and grow our careers as pathologists;

\section{ACKNOWLEDGEMENT}

We would like to thank all pathologists of FESPATH group whatsup for allowing us to publish this study. We also thank Khanoussi Wafa and Marwa Atmane for the English editing of this paper.

\section{CONFLICT of INTEREST}

The authors declare no conflict of interest.

\section{REFERENCES}

1. Oltulu P, Mannan AASR, Gardner JM. Effective use of Twitter and Facebook in pathology practice. Hum Pathol. 2018;73:12843.

2. Sarode SC, Sarode GS, Anand R, Patil S, Unadkat H. WhatsApp is an effective tool for obtaining second opinion in oral pathology practice. J Oral Pathol Med. 2017;46:513-9.

3. Mars M, Scott RE. WhatsApp in clinical practice: A literature. Stud Health Technol Inform. 2016;231:82-90.

4. Khanna V, Sambandam SN, Gul A, Mounasamy V. "WhatsApp" ening in orthopedic care: A concise report from a 300-bedded tertiary care teaching center. Eur J Orthop Surg Traumatol. 2015;25:821-6.

5. Zotti F, Dalessandri D, Salgarello S, Piancino M, Bonetti S, Visconti L, Paganelli C. Usefulness of an app in improving oral hygiene compliance in adolescent orthodontic patients. Angle Orthod. 2016;86:101-7.

6. Petruzzi M, De Benedittis M. WhatsApp: A telemedicine platform for facilitating remote oral medicine consultation and improving clinical examinations. Oral Surg Oral Med Oral Pathol Oral Radiol. 2016;121:248-54.

7. Goyal A, Tanveer N, Sharma P. WhatsApp for teaching pathology postgraduates: A pilot study. J Pathol Inform. 2017;8:6.

8. Fuller MY, Allen TC. Let's have a Tweetup: The case for using Twitter professionally. Arch Pathol Lab Med. 2016;140:956-7.

9. Fuoco M, Leveridge MJ. Early adopters or laggards? Attitudes toward and use of social media among urologists. BJU Int. 2015;115:491-7.

10. Salem J, Borgmann H, Murphy DG. Integrating social media into urologic health care: What can we learn from other disciplines? Curr Urol Rep. 2016;17(2):13.

11. Isom J, Walsh M, Gardner JM. Social media and pathology: Where are we now and why does it matter? Adv Anat Pathol. 2017;24:294-303.

12. Gonzalez RS, Amer SM, Yahia NB, Costa FD, Noatay M, Qiao JH, Rosado FG, Rosen Y, Sedassari BT, Yantiss RK, Gardner JM. Facebook discussion groups provide a robust worldwide platform for free pathology education. Arch Pathol Lab Med. 2017;141:690-5.

13. Crane GM, Gardner JM. Pathology image-sharing on social media: Recommendations for protecting privacy while motivating education. AMA J Ethics. 2016;18:817-25. 